\title{
A Study on the Inj-Equitable Graph of a Graph
}

\author{
Hanaa Alashwali ${ }^{*}$, Ahmad N. Alkenani' ${ }^{1}$ A. Saleh ${ }^{2}$, Najat Muthana1 \\ ${ }^{1}$ Department of Mathematics, King Abdulaziz University, Jeddah, KSA \\ ${ }^{2}$ Department of Mathematics, Jeddah University, Jeddah, KSA \\ Email: ${ }^{*}$ halashwali@kau.edu.sa
}

How to cite this paper: Alashwali, H., Alkenani, A.N., Saleh, A. and Muthana, N. (2018) A Study on the Inj-Equitable Graph of a Graph. Applied Mathematics, 9, 264-273. https://doi.org/10.4236/am.2018.93020

Received: February 13, 2018

Accepted: March 26, 2018

Published: March 29, 2018

Copyright $\odot 2018$ by authors and Scientific Research Publishing Inc. This work is licensed under the Creative Commons Attribution International License (CC BY 4.0).

http://creativecommons.org/licenses/by/4.0/

\section{Abstract}

For any graph $G$, the Inj-equitable graph of a graph $G$, denoted by $I E(G)$, is the graph with the same vertices as $G$ and for any two adjacent vertices $u$ and $v$ in $I E(G),\left|\operatorname{deg}_{\text {in }}(u)-\operatorname{deg}_{\text {in }}(v)\right| \leq 1$, where for any vertex $w \in V(G)$, $\operatorname{deg}_{\text {in }}(w)=\left|\left\{w^{\prime} \in V: N\left(w^{\prime}\right) \cap N(w) \neq \phi\right\}\right|$. In this paper, Inj-equitable graphs of some graphs are obtained, and some properties and results are established. Moreover, complete Inj-equitable graph and the Inj-equitable graph are defined.

\section{Keywords}

Domination, Injective Equitable Graph, Equitable Matrix, Injective Matrix

\section{Introduction}

We consider only finite undirected graphs $G=(V, E)$ without loops and multiple edges. $\langle X\rangle$ will denote the subgraph of $G$ induced by a set of vertices $X$. For any vertex $v \in V(G)$ the open neighborhood of $v$ is the set $N(v)=\{u \in V(G): u v \in E\}$. The closed neighborhood of $v$ is $N[v]=N(v) \cup v$. The degree of a vertex $v$ in $G$ is $\operatorname{deg}(v)=|N(v)| . \Delta(G)$ and $\delta(G)$ are the maximum and minimum vertex degree of $G$ respectively. The distance $d(u, v)$ between any two vertices $u$ and $v$ in a graph $G$ is the number of the edges in a shortest path. The eccentricity of a vertex $u$ in a connected graph $G$ is $e(u)=\max \{d(u, v), v \in V\}$. The diameter of $G$ is the value of the greatest eccentricity, and the radius of $G$ is the value of the smallest eccentricity. The clique number of $G$, denoted by $\omega(G)$, is the order of the maximal complete subgraph of $G$. The Inj-neighborhood of a vertex $u \in V(G)$ denoted by $N_{\text {in }}(u)$ is defined as $N_{\text {in }}(u)=\{v \in V(G):|\Gamma(u, v)| \geq 1\}$, where $|\Gamma(u, v)|$ is the number of common neighborhood between the vertices $u$ and $v$. The cardinality 
of $N_{\text {in }}(u)$ is called injective degree of the vertex $u$ and is denoted by $\operatorname{deg}_{\text {in }}(u)$ in $G$. The corona product $G \circ H$ is obtained by taking one copy of $G$ and $V(G) \mid$ copies of $H$ and by joining each vertex of the $i$-th copy of $H$ to the $i$-th vertex of $G$, where $1 \leq i \leq|V(G)|$. The cartesian product $G \times H$ is a graph with vertex set $V(G) \times V(H)$ and edge set $E(G \times H)=\left\{\left(\left(u, u^{\prime}\right),\left(v, v^{\prime}\right)\right): u=v\right.$ and $\left(u^{\prime}, v^{\prime}\right) \in E(H)$, or $u^{\prime}=v^{\prime}$ and $\left.(u, v) \in E(G)\right\}$. For more terminologies and notations, we refer the reader to [1] [2] [3] and [4].

The common neighborhood graph (congraph) of a graph $G$, denoted by $\operatorname{con}(G)$, is the graph with vertex set $\left\{v_{1}, v_{2}, \cdots, v_{n}\right\}$, in which two vertices are adjacent if and only if they have at least one common neighbor in the graph $G$ [5]. The equitable graph of a graph $G$, denoted by $G^{e}$, is the graph with vertex set $V(G)$ and two vertices $u$ and $v$ are adjacent if and only if $|\operatorname{deg}(u)-\operatorname{deg}(v)| \leq 1 \quad[6]$.

\section{IE-Graph of a Graph}

The Inj-equitable dominating sets on graphs which introduced in [7] motivated us to define two new graphs: the IE-graph of a graph and the IE-graph. In this section, we define the Inj-equitable graph of a graph and study some properties of this graph. Also, the injective equitable graph of some graph's families are found.

Definition 1 Let $G=(V, E)$ be a graph. The injective equitable (Inj-equitable) graph of a graph $G$, denoted by $\operatorname{IE}(G)$, is defined as the graph with the same vertices as $G$ and two vertices $u$ and $v$ are adjacent in $\operatorname{IE}(G)$ if

$$
\left|\operatorname{deg}_{\text {in }}(u)-\operatorname{deg}_{\text {in }}(v)\right| \leq 1 \text {. }
$$

Example 2 Let $G$ be a graph as in Figure 1. Then, $I E(G) \cong K_{7} \cup K_{5} \cup K_{2}$.

The Inj-equitable graph of some known graphs are given in the following observation:

\section{Observation 3}

(i) For any path $P_{n}$ with $n$ vertices, $\operatorname{IE}\left(P_{n}\right) \cong K_{n}$.

(ii) For any cycle $C_{n}$ with $n$ vertices, $\operatorname{IE}\left(C_{n}\right) \cong K_{n}$.

(iii) For any complete graph $K_{n}, \operatorname{IE}\left(\bar{K}_{n}\right)=\operatorname{IE}\left(K_{n}\right)=K_{n}$.

Proposition 4 For any complete bipartite graph $K_{r, s}$,

$$
\operatorname{IE}\left(K_{r, s}\right) \cong \begin{cases}K_{r+s} & \text { if }|r-s| \leq 1 ; \\ K_{r} \cup K_{s} & \text { otherwise. }\end{cases}
$$

Proof. Let $G \cong K_{r, s}$ be a complete bipartite graph with partite sets $A$ and $B$ such that $|A|=r,|B|=s$. Clearly for any vertex $v$ from $A, \operatorname{deg}_{i n}(v)=r-1$ and for any vertex $u$ from $B, \operatorname{deg}_{i n}(u)=s-1$. Therefore, for any two vertices $u$ and $v$ in $K_{r, s},\left|\operatorname{deg}_{i n}(u)-\operatorname{deg}_{i n}(v)\right|=|r-s|$. If $|r-s| \leq 1$, then $\operatorname{IE}\left(K_{r, s}\right)=K_{r+s}$. Otherwise, $I E\left(K_{r, s}\right)=K_{r} \cup K_{s}$.

We will generalize Proposition 4 in the following result.

Proposition 5 For any multipartite graph $K_{n_{1}, n_{2}, \cdots, n_{m}}$, where $m \geq 3$, 


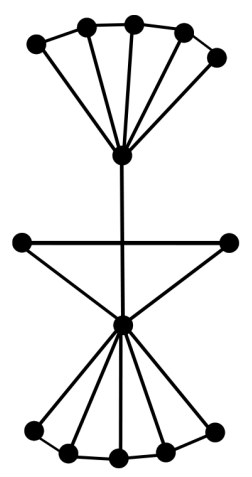

Figure 1. A graph $G$.

$\operatorname{IE}\left(K_{n_{1}, n_{2}, \cdots, n_{m}}\right)=K_{r}$, where $r=\sum_{i=1}^{m} n_{i}$.

Proof. Let $G$ be a multipartite graph $K_{n_{1}, n_{2}, \cdots, n_{m}}$ where $m \geq 3$. Then for every vertex $u$ in $G, \operatorname{deg}_{i n}(u)=n_{1}+n_{2}+\cdots+n_{m}-1$. Hence, $\operatorname{IE}\left(K_{n_{1}, n_{2}, \cdots, n_{m}}\right)=K_{r}$, where $r=\sum_{i=1}^{m} n_{i}$.

Bi-star graph is the graph obtained by joining the apex vertices of two copies of star $K_{1, n}$.

Proposition 6 For any bi-star graph $B(s, t)$,

$$
\operatorname{IE}(B(s, t))= \begin{cases}K_{s+t+2} & \text { if }|s-t| \leq 1 ; \\ K_{s+1} \cup K_{t+1} & \text { otherwise. }\end{cases}
$$

Proof. Let $G \cong B(s, t)$ be a bi-star graph labeling as in Figure 2. Therefore, $\operatorname{deg}_{\text {in }}(u)=t, \quad \operatorname{deg}_{\text {in }}(v)=s, \quad$ for $i=1,2, \cdots, s, \quad \operatorname{deg}_{\text {in }}\left(u_{i}\right)=s \quad$ and for $j=1,2, \cdots, t, \operatorname{deg}_{\text {in }}\left(v_{j}\right)=t$. So, if $|s-t| \leq 1$, then $\operatorname{IE}(B(s, t))=K_{s+t+2}$. Otherwise, $\operatorname{IE}(B(s, t))=K_{s+1} \cup K_{t+1}$.

A firefly graph $F_{r, s, t}$ is a graph on $2 r+2 s+t+1$ vertices that consists of $r$ triangles, $s$ pendant paths of length 2 and $t$ pendant edges sharing a common vertex.

Proposition 7 For any firefly graph $F_{r, s, t}, \quad r \geq 1, s \geq 1$ and $t \geq 1$,

$$
I E\left(F_{r, s, t}\right)= \begin{cases}K_{2 r+s+2} \cup K_{s} & \text { if } t=1 ; \\ K_{2 r+s+2} \cup K_{1, s+2} \cup K_{s} & \text { if } t=2 ; \\ K_{2 r+s+t} \cup K_{1} \cup K_{s} & \text { if } t>2 .\end{cases}
$$

Proof. Let $G$ be a firefly graph $F_{r, s, t}$ as in Figure 3, where $r \geq 1, s \geq 1$ and $t \geq 1$. Let $v$ be the center vertex, $v_{i}, i=1,2, \cdots, 2 r$ be any vertex from the triangle other than $v . w_{i}, i=1,2, \cdots, t$ be any end vertex in the pendant edge. $u_{i}$ and $u_{i}^{\prime}, i=1,2, \cdots, s$ be any end vertex and internal vertex respectively in the pendant path. Then, $\operatorname{deg}_{\text {in }}(v)=2 r+s, \operatorname{deg}_{\text {in }}\left(v_{i}\right)=2 r+s+t$, $\operatorname{deg}_{\text {in }}\left(w_{i}\right)=2 r+s+t-1, \operatorname{deg}_{\text {in }}\left(u_{i}^{\prime}\right)=2 r+s+t-1$ and $\operatorname{deg}_{\text {in }}\left(u_{i}\right)=1$. There are three cases:

Case 1. Suppose that $t=1$. Then, $\left|\operatorname{deg}_{\text {in }}(v)-\operatorname{deg}_{\text {in }}\left(v_{i}\right)\right|=1$, $\mid \begin{aligned} & \operatorname{deg}_{\text {in }}(v)-\operatorname{deg}_{\text {in }}\left(w_{i}\right)|=0,| \operatorname{deg}_{\text {in }}(v)-\operatorname{deg}_{\text {in }}\left(u_{i}^{\prime}\right) \mid=0, \\ & \operatorname{deg}_{\text {in }}\left(v_{i}\right)-\operatorname{deg}_{\text {in }}\left(w_{i}\right)|=1,| \operatorname{deg}_{\text {in }}\left(v_{i}\right)-\operatorname{deg}_{\text {in }}\left(u_{i}^{\prime}\right) \mid=1 \text { and }\end{aligned}$ 


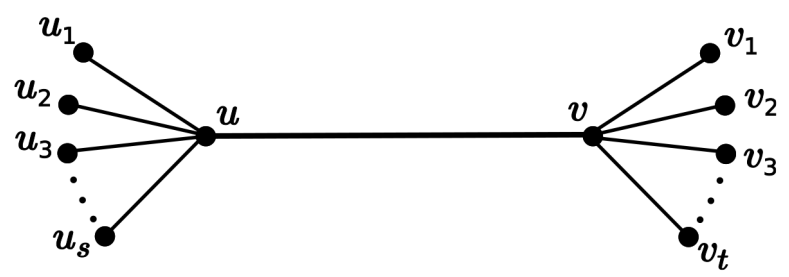

Figure 2. A bi-star graph.

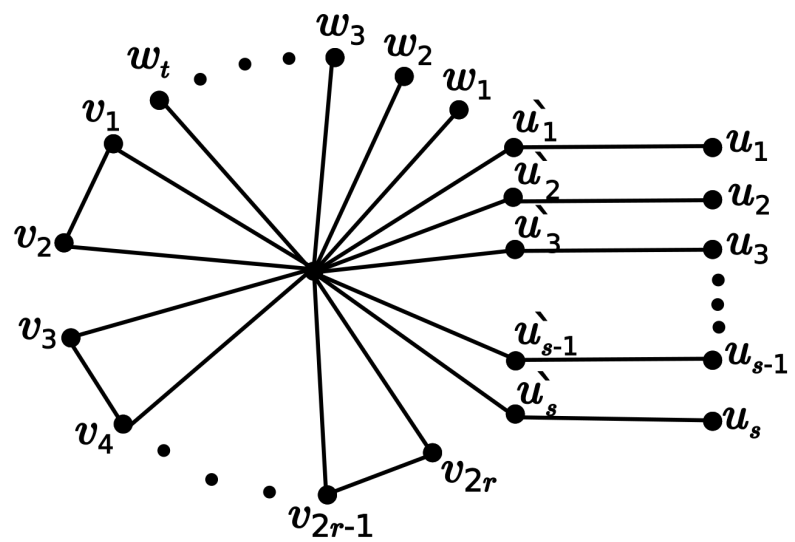

Figure 3. A firefly graph.

$\left|\operatorname{deg}_{\text {in }}\left(w_{i}\right)-\operatorname{deg}_{\text {in }}\left(u_{i}^{\prime}\right)\right|=0$. Hence, $I E\left(F_{r, s, t}\right) \cong K_{2 r+s+2} \cup K_{s}$.

Case 2. Suppose that $t=2$. Then, $\left|\operatorname{deg}_{\text {in }}(v)-\operatorname{deg}_{\text {in }}\left(w_{i}\right)\right|=1$, $\left|\operatorname{deg}_{\text {in }}(v)-\operatorname{deg}_{\text {in }}\left(u_{i}^{\prime}\right)\right|=1,\left|\operatorname{deg}_{\text {in }}\left(v_{i}\right)-\operatorname{deg}_{\text {in }}\left(w_{i}\right)\right|=1$, $\left|\operatorname{deg}_{\text {in }}\left(v_{i}\right)-\operatorname{deg}_{\text {in }}\left(u_{i}^{\prime}\right)\right|=1$ and $\left|\operatorname{deg}_{\text {in }}\left(w_{i}\right)-\operatorname{deg}_{\text {in }}\left(u_{i}^{\prime}\right)\right|=0$.

Hence $I E\left(F_{r, s, t}\right) \cong K_{2 r+s+2} \cup K_{1, s+2} \cup K_{s}$.

Case 3. Suppose that $t \leq 2$. Then, $\left|\operatorname{deg}_{\text {in }}\left(v_{i}\right)-\operatorname{deg}_{\text {in }}\left(w_{i}\right)\right|=1$, $\left|\operatorname{deg}_{\text {in }}\left(v_{i}\right)-\operatorname{deg}_{\text {in }}\left(u_{i}^{\prime}\right)\right|=1$ and $\left|\operatorname{deg}_{\text {in }}\left(w_{i}\right)-\operatorname{deg}_{\text {in }}\left(u_{i}^{\prime}\right)\right|=0$.

Hence $\operatorname{IE}\left(F_{r, s, t}\right) \cong K_{2 r+s+t} \cup K_{1} \cup K_{s}$.

Definition 8 A complete chain graph denoted by $C C\left(n_{1}, s_{1}, n_{2}, s_{2}, \cdots, s_{r-1}, n_{r}\right)$ is a chain of complete graphs $K_{n_{1}}, K_{n_{2}}, \cdots, K_{n_{r}}$ such that there are $s_{i}$ common vertices between $K_{n_{i}}$ and $K_{n_{i+1}}$, for $i=1,2, \cdots, r-1$.

Proposition 9 Let $G$ be a graph such that $G \cong P_{m} \times P_{2}$. Then

$$
I E(G)= \begin{cases}K_{2 m} & \text { if } m \leq 4 \\ C C(8,4,2 m-4) & \text { if } m \geq 5 .\end{cases}
$$

Proof. Let $G$ be a graph such that $G \cong P_{m} \times P_{2}$. Then there are two cases:

Case 1. If $m \leq 4$, for all $v \in V(G)$, either $\operatorname{deg}_{\text {in }}(v)=2$ or $\operatorname{deg}_{\text {in }}(v)=3$. Therefore, for any two vertices $u$ and $v,\left|\operatorname{deg}_{\text {in }}(u)-\operatorname{deg}_{\text {in }}(v)\right| \leq 1$. Hence, $I E(G) \cong K_{2 m}$.

Case 2. If $m \geq 5$, there are 4 vertices with Inj-degree 2,4 vertices with Inj-degree 3 and $2 m-8$ vertices with Inj-degree 4 . Therefore,

$$
\operatorname{IE}(G) \cong C C(8,4,2 m-4) .
$$

Proposition 10 Let $G$ be a graph such that $G \cong P_{m} \times P_{3}$, where $m \geq 4$. Then 


$$
I E(G)= \begin{cases}C C(10,4,6) & \text { if } m=4 \\ C C(10,4,2 m-2,2 m-6,3 m-10) & \text { if } m \geq 5\end{cases}
$$

Proof. Suppose that, $G \cong P_{m} \times P_{3}$. Then we have two cases:

Case 1. If $m=4$, then there are 6 vertices have Inj-degree 3, 4 vertices have Inj-degree 4 and 2 vertices have Inj-degree 5 . Therefore, $I E(G) \cong C C(10,4,6)$.

Case 2. If $m \geq 5$, then there are 6 vertices have Inj-degree 3, 4 vertices have Inj-degree $4,2 m-6$ vertices have Inj-degree 5 and $m-4$ vertices of degree 6 . Therefore, $\operatorname{IE}(G) \cong C C(10,4,2 m-2,2 m-6,3 m-10)$.

Theorem 11 Let $G$ be a graph such that $G \cong P_{m} \times P_{n}$, where $m, n \geq 5$. Then $I E(G) \cong C C(12,8,2 m+2 n-4,2 m+2 n-12,2 m+2 n-8,4,2 m+2 n-8,2 m+2 n-12$, $(n-2)(m-2))$.

Proof. Suppose that $G \cong P_{m} \times P_{n}$. Therefore, there are 4 vertices have Inj-degree 3, 8 vertices have Inj-degree $4,2 m+2 n-12$ have Inj-degree 5,4 vertices have Inj-degree $6,2 m+2 n-12$ have Inj-degree 7 and $(n-4)(m-4)$ have Inj-degree 8 . Hence $\operatorname{IE}\left(P_{m} \times P_{n}\right) \cong C C(12,8,2 m+2 n-4,2 m+2 n-12$, $2 m+2 n-8,4,2 m+2 n-8,2 m+2 n-12,(n-2)(m-2))$.

The generalized Petersen graph $G P(m, n)$ is defined by taking

$$
V(G P(m, n))=\left\{u_{i}, v_{i}: 0 \leq i \leq m-1\right\}
$$

and

$$
E(G P(m, n))=\left\{u_{i} u_{i+1}, u_{i} v_{i}, v_{i} v_{i+n}: 0 \leq i \leq m-1\right\}
$$

where the subscripts are integers modulo $m, m \geq 5$ and $1 \leq n \leq\left\lfloor\frac{m-1}{2}\right\rfloor$.

Theorem 12 Let $G$ be a generalized Petersen graph $G P(m, n)$. Then, $\operatorname{IE}(G) \cong K_{m+n}$.

Proof. Let $G$ be a generalized Petersen graph $G P(m, n)$ with vertices as in Figure 4. Then, $N_{i n}\left(u_{i}\right)=\left\{u_{i+2}, u_{i-2}, v_{i+n}, v_{i-n}, v_{i-1}, v_{i+1}\right\}$ and $N_{i n}\left(v_{i}\right)=\left\{u_{i+1}, u_{i-1}, u_{i+n}, u_{i-n}, v_{i+2 n}, v_{i-2 n}\right\}$. Therefore, for $0 \leq i \leq m-1$, $\operatorname{deg}_{\text {in }}\left(u_{i}\right)=\operatorname{deg}_{\text {in }}\left(v_{i}\right)=6$. Hence, $\operatorname{IE}(G) \cong K_{m+n}$.

Proposition 13 Let $G \cong C_{m} \times P_{3}$. Then $I E(G) \cong K_{3 m}$.

Proof. Let $G \cong C_{m} \times P_{3}$. We have three cases:

Case 1. If $m=3$, then for all $v \in V(G), \operatorname{deg}_{\text {in }}(v)=5$. Therefore, all the vetrices have the same Inj-degree. Hence, $\operatorname{IE}(G) \cong K_{3 m}$.

Case 2. If $m=4$, then for all $v \in V(G), \operatorname{deg}_{\text {in }}(v)=4$ or 5 . Therefore, $I E(G) \cong K_{3 m}$.

Case 3. If $m \geq 5$, then for all $v \in V(G), \operatorname{deg}_{\text {in }}(v)=5$ or 6 . Therefore, for any two vertices $u$ and $v$ in $G$,

$$
\left|\operatorname{deg}_{\text {in }}(u)-\operatorname{deg}_{\text {in }}(v)\right|=1 \text {. Hence, } \operatorname{IE}(G) \cong K_{3 m} \text {. }
$$

Theorem 14 For any graph $G$ such that $G \cong C_{m} \times P_{n}$,

$$
\operatorname{IE}(G) \cong K_{2 m} \cup K_{m(n-2)} \text {, where } n \geq 5 \text {. }
$$

Proof. Suppose that $G \cong C_{m} \times P_{n}$. Then we have $2 m$ vertices of injective degree $5,2 m$ vertices of injective degree 7 and $m(n-4)$ vertices of injective degree 8. Hence, $I E(G) \cong K_{2 m} \cup K_{m(n-2)}$. 


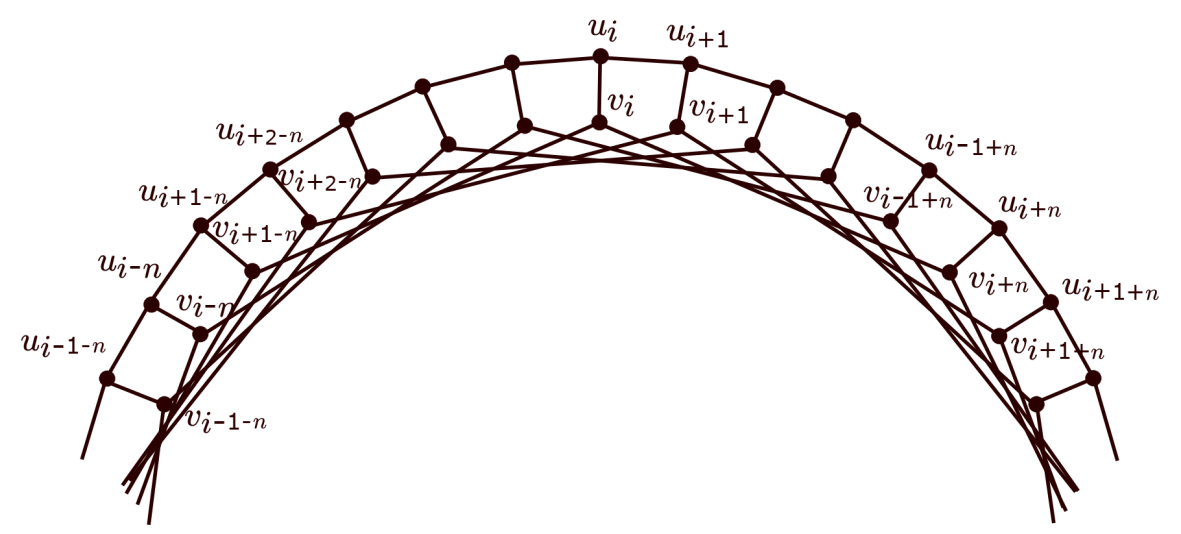

Figure 4. Generalized Petersen graph $G P(m, n)$.

Proposition 15 For any graph $G$, such that $G \cong C_{n} \times C_{m}, \quad I E(G) \cong K_{n m}$.

Proof. Let $G$ be a graph such that $G \cong C_{n} \times C_{m}$. Then every vertex in $G$ has injective degree 8. Hence, $I E(G) \cong K_{n m}$.

Proposition $16 I E\left(K_{n} \circ \bar{K}_{m}\right)=K_{n} \cup K_{n m}$.

Proof. Consider the corona $K_{n} \circ \bar{K}_{m}$. The interior vertices have injective degree $(n-1)(m+1)$ and the outer vertices have injective degree $n+m-2$. Therefore $I E\left(K_{n} \circ \bar{K}_{m}\right)=K_{n} \cup K_{n m}$.

Theorem 17 For any graph $G$ with $\delta \geq 2$, if $G$ is $k$-regular or $(k, k+1)$ biregular, then

$$
I E\left(G \circ \bar{K}_{m}\right)=K_{n} \cup K_{m n},
$$

where $n$ is the number of vertices in $G$.

Proof. Let $G$ be a $k$-regular graph with $n$ vertices and $\delta \geq 2$. Suppose that $\left\{u_{1}, u_{2}, \cdots, u_{n}\right\}$ and $\left\{v_{1}, v_{2}, \cdots, v_{m}\right\}$ are the vertex sets of $G$ and $\bar{K}_{m}$, respectively. Therefore, for $i=1,2, \cdots, n, \operatorname{deg}_{i n}\left(u_{i}\right)=k(m+1)$ and for $j=1,2, \cdots, m$, $\operatorname{deg}_{\text {in }}\left(v_{j}\right)=k+m-1$. Therefore, $\left|\operatorname{deg}_{i n}\left(u_{i}\right)-\operatorname{deg}_{\text {in }}\left(v_{j}\right)\right|=m(k-1)+1>1$. Hence, $I E\left(G \circ \bar{K}_{m}\right)=K_{n} \cup K_{m n}$. Similarly, we can prove if $G$ is $(k, k+1)$-biregular, then $I E\left(G \circ \bar{K}_{m}\right)=K_{n} \cup K_{m n}$.

Proposition 18 For any cycle graph $C_{n}$, IE $\left(C_{n} \circ K_{1}\right)=2 K_{n}$.

Proof. Let $u_{i}$ be any vertex on the cycle $C_{n}$ and $v_{i}$ be any vertex outside the cycle $C_{n}$. Then $\operatorname{deg}_{i n}\left(u_{i}\right)=4$ and $\operatorname{deg}_{i n}\left(v_{i}\right)=4$. Therefore, $\operatorname{IE}\left(C_{n} \circ K_{1}\right)=2 K_{n}$.

Definition 19 Let $G=(V, E)$ be a graph. A subset $D$ of $V$ is called degree Inj-equitable set if the difference between the injective degree of any two vertices in $D$ less then or equal one. The maximum cardinality of a degree Inj-equitable set in $G$ is called degree Inj-equitable number of $G$ and is denoted by $D_{i e}(G)$. the minimum cardinality of a maximal degree Inj-equitable set is called the lower degree Inj-equitable number of $G$ and is denoted by $d_{i e}(G)$.

Observation 20 For any integer $\delta \leq i \leq \Delta-1$, let $S_{i}=\left\{v \in V: \operatorname{deg}_{i n}(v)=i\right.$ or $i+1\}$. A nonempty subset $A$ of $V$ is a maximal degree Inj-equitable if and only if $A=S_{i}$ for some i. Hence, $D_{i e}(G)=\max \left\{\left|S_{i}\right|: \delta \leq i \leq \Delta-1\right.$ and $\left.S_{i} \neq 0\right\}$ and 
$d_{i e}(G)=\min \left\{\left|S_{i}\right|: \delta \leq i \leq \Delta-1\right.$ and $\left.S_{i} \neq 0\right\}$.

Observation 21 Let $G=(V, E)$ be a graph. The intersection graph of maximal degree Inj-equitable sets denoted by $G_{i n}$ is defined on the family of all maximal degree Inj-equitable sets of $G$ and has the property that any two vertices are adjacent if their intersection is not empty.

Theorem 22 For any graph $G$

$$
\Delta(I E(G))=D_{\text {ie }}(G)-1
$$

and

$$
\delta(I E(G))=d_{i e}(G)-1 .
$$

Proof. Since $\left\langle S_{i}\right\rangle$ is complete subgraph in $I E(G)$ and from Observation $20 D_{i e}(G)=\max \left\{\left|S_{i}\right|: \delta \leq i \leq \Delta-1\right.$ and $\left.S_{i} \neq 0\right\}$, then $D_{i e}(G)$ is the order of the maximum complete subgraph in $I E(G)$. Therefore, for any vetrex in the maximum complete subgraph has degree $D_{i e}(G)-1$. Hence $\Delta(I E(G))=D_{i e}(G)-1$.

Similarly, we can show that $\delta(\operatorname{IE}(G))=d_{i e}(G)-1$.

The following proposition can be proved straightforward.

Proposition 23 For any graph $G$,

(i) $\operatorname{diam}(\operatorname{IE}(G))=\Delta_{\text {in }}(G)-\delta_{\text {in }}(G)$.

(ii) $\omega(\operatorname{IE}(G))=D_{\text {ie }}(G)$, where $\omega(\operatorname{IE}(G))$ is the clique number of $\operatorname{IE}(G)$.

Theorem 24 Let $G$ be any graph. The number of edges in $I E(G)$ is given by:

$$
\sum_{i=\delta}^{\Delta-1} \frac{\left|S_{i}\right|\left(\left|S_{i}\right|-1\right)}{2}-\sum_{i=\delta}^{\Delta-1} \frac{\left|S_{i} \cap S_{i+1}\right|\left(\left|S_{i} \cap S_{i-1}\right|-1\right)}{2}
$$

where $S_{i}=\left\{v \in V: \operatorname{deg}_{\text {in }}(v)=i\right.$ or $\left.i+1\right\}$.

Proof. Let $G$ be any graph. Since each $\left\langle S_{i}\right\rangle$ is complete subgraph graph in $\operatorname{IE}(G)$, then $\left\langle S_{i}\right\rangle$ has $\frac{\left|S_{i}\right|\left(\left|S_{i}\right|-1\right)}{2}$ edges. But the edges in $\left\langle S_{i+1} \cap S_{i}\right\rangle$ are counted twice in $\frac{\left|S_{i}\right|\left(\left|S_{i}\right|-1\right)}{2}$. Hence the number of edges in $I E(G)$ is given by

$$
\sum_{i=\delta}^{\Delta-1} \frac{\left|S_{i}\right|\left(\left|S_{i}\right|-1\right)}{2}-\sum_{i=\delta}^{\Delta-1} \frac{\left|S_{i} \cap S_{i+1}\right|\left(\left|S_{i} \cap S_{i-1}\right|-1\right)}{2} .
$$

Theorem 25 Let $G$ be any graph. Then the following are equivalent:

(i) $I E(G)$ is connected graph.

(ii) The distinct sequence of the Inj-equitable degrees are $\delta_{\text {in }}(G), \delta_{i}(G)+1, \delta_{\text {in }}(G)+2, \cdots, \Delta_{\text {in }}(G)$.

(iii) The intersection graph of maximal degree Inj-equitable sets $G_{i n}$ is a path.

Proof. (i) $\Rightarrow$ (ii): Assume to the contrary, that $I E(G)$ is connected and there exist an integer $\delta_{i n}(G) \leq i \leq \Delta_{i n}(G)$. Then $S_{i}$ and $S_{i-1}$ has no common vertices, that means $\operatorname{IE}(G)$ is not connected which is a contradiction. Hence, the distinct sequence of the Inj-equitable degrees are

$$
\delta_{\text {in }}(G), \delta_{i}(G)+1, \delta_{\text {in }}(G)+2, \cdots, \Delta_{\text {in }}(G) \text {. }
$$


(ii) $\Rightarrow$ (iii): Suppose that the distinct sequence of the Inj-equitable degrees are $\delta_{\text {in }}(G), \delta_{\text {in }}(G)+1, \delta_{\text {in }}(G)+2, \cdots, \Delta_{\text {in }}(G)$. Then $S_{i} \cap S_{i+1} \neq \phi$ and $S_{i} \cap S_{j}=\phi$ if $|i-j| \geq 2$. Hence, the intersection graph of maximal degree Inj-equitable sets $G_{\text {in }}$ is a path.

(iii) $\Rightarrow(\mathrm{i})$ : Suppose that $G_{i n}$ is a path. Then $S_{i} \cap S_{i+1} \neq \phi$. Since $\left\langle S_{i}\right\rangle$ is complete, then $\operatorname{IE}(G)$ is connected.

Proposition 26 For any graph $G, I E(G)$ is totally disconnected if and only if $G \cong K_{1}$.

Proof. Let $G$ be a graph such that $\operatorname{IE}(G)$ is totally disconnected. Therefore, $\Delta(\operatorname{IE}(G))=\delta(\operatorname{IE}(G))=0$. So, from Theorem 22, $D_{i e}(G)-1=d_{i e}(G)-1=0$. Therefore, $D_{\text {ie }}(G)=d_{\text {ie }}(G)=1$. Hence, $G \cong K_{1}$.

Conversely, suppose that $G \cong K_{1}$. Therefore, $G$ has only one maximal degree Inj-equitable set of order one. So, $D_{i e}(G)=d_{i e}(G)=1$. Therefore, $\Delta(\operatorname{IE}(G))=\delta(\operatorname{IE}(G))=0$. Hence, $\operatorname{IE}(G)$ is totally disconnected.

Theorem 27 For any graph $G$ with $n$ vertices, $\operatorname{IE}(G) \cong K_{n}$ if and only if $G$ has only one maximal degree Inj-equitable set.

Proof. Let $G$ be a graph of order $n$ and $I E(G) \cong K_{n}$. Therefore, for any vertices $u$ and $v$ in $G,\left|\operatorname{deg}_{\text {in }}(u)-\operatorname{deg}_{\text {in }}(v)\right| \leq 1$. Hence, $G$ has only one maximal degree Inj-equitable set. Conversely, suppose that $G$ has only one maximal degree Inj-equitable set $S_{i}$. Since $\left\langle S_{i}\right\rangle$ is complete in $\operatorname{IE}(G)$, then $\operatorname{IE}(G) \cong K_{n}$.

Next theorem gives the relation between the $I E(G), \operatorname{Con}(G)$ and $G^{e}$.

Theorem 28 For any graph $G, \operatorname{IE}(G) \cong(\operatorname{con}(G))^{e}$.

Proof. Let $G$ be any graph and consider $\operatorname{con}(G)$ and $I E(G)$. Both graphs have the same vertices. Now, let $f=u v$ be any edge in $\operatorname{IE}(G)$. Then, $\left|\operatorname{deg}_{\text {in }}(u)-\operatorname{deg}_{\text {in }}(v)\right| \leq 1$ in $G$. Since $\operatorname{deg}_{\text {in }}(u)$ in $G$ is equal $\operatorname{deg}(u)$ in $\operatorname{con}(G)$, then, $|\operatorname{deg}(u)-\operatorname{deg}(v)| \leq 1$ in $\operatorname{con}(G)$. Therefore, $f$ is an edge in $(\operatorname{con}(G))^{e}$. Hence $\operatorname{IE}(G)$ is a subgraph of $(\operatorname{con}(G))^{e}$.

Similarly we can show that $(\operatorname{con}(G))^{e}$ is a subgraph of $\operatorname{IE}(G)$.

\section{Injective Equitable Graphs}

Definition 29 A graph $G$ is said to be injective equitable graph (IE-graph) if there exists a graph $H$ such that $I E(H) \cong G$.

For example, any complete graph is $I E$-graph.

Definition 30 The family of graphs $H$ which satisfy the condition $\operatorname{IE}(H) \cong G$ is called the injective equitable family of $G$ and denoted by $G_{I E}$. i.e,

$$
G_{I E}=\{H: I E(H) \cong G\} .
$$

Example 31 Let $H_{1}, H_{2}$ and $H_{3}$ be figures as an Figure 5. Then, $\operatorname{IE}\left(H_{i}\right)=K_{3}$ for all $i=1,2,3$.

Lemma 32 Any path $P_{n}$ is not IE-graph.

Proof. Suppose, to the contrary, that $G \cong P_{n}$ such that $G$ is $I E$-graph. Therefore, there exist a graph $H$ such that $\operatorname{IE}(H) \cong G$. Let $v_{1}, v_{2}$ and $v_{3}$ be 

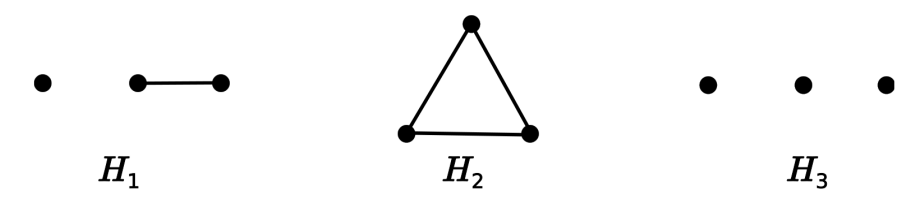

Figure 5. $I E\left(H_{i}\right)=K_{3}$.

any vertices in $G$ such that $v_{1}$ adjacent to $v_{2}$ and $v_{2}$ adjacent to $v_{3}$. Suppose that $\operatorname{deg}_{\text {in }}\left(v_{1}\right)=i$ in $H$. Therefore, $\operatorname{deg}_{\text {in }}\left(v_{2}\right)=i-1$ or $i$ or $i+1$.

Case 1. $\operatorname{deg}_{\text {in }}\left(v_{2}\right)=i-1$. Therefore, $\operatorname{deg}_{\text {in }}\left(v_{3}\right)=i-2$ or $i-1$ or $i$. If $\operatorname{deg}_{\text {in }}\left(v_{3}\right)=i-1$ or $i$, then $v_{1}$ and $v_{3}$ are adjacent which contradicts that $G$ is a path. So, $\operatorname{deg}_{\text {in }}\left(v_{3}\right)=i-2$.

Case 2. $\operatorname{deg}_{\text {in }}\left(v_{2}\right)=i$. Therefore, $\operatorname{deg}_{\text {in }}\left(v_{3}\right)=i-1$ or $i$ or $i+1$. So, $v_{1}$ and $v_{3}$ are adjacent which contradicts that $G$ is a path. Hence, $\operatorname{deg}_{i n}\left(v_{2}\right) \neq i$.

Case 3. $\operatorname{deg}_{\text {in }}\left(v_{2}\right)=i+1$. Therefore, $\operatorname{deg}_{\text {in }}\left(v_{3}\right)=i$ or $i+1$ or $i+2$. If $\operatorname{deg}_{\text {in }}\left(v_{3}\right)=i$ or $i+1$, then $v_{1}$ and $v_{3}$ are adjacent which contradicts that $G$ is a path. So, $\operatorname{deg}_{\text {in }}\left(v_{3}\right)=i+2$.

Hence the graph $H$ has different injective degrees which contradicts that any graph with $n$ vertices has at least two vertices of the same Inj-degree. Therefore, $P_{n}$ is not $I E$-graph.

Lemma 33 Any cycle $C_{n}$ is not IE-graph, where $n \geq 4$.

Proof. Suppose, to the contrary, that $G$ is a cycle such that $G$ is $I E$-graph. Therefore, there exist a graph $H$ such that $I E(H) \cong G$. Let $v_{1}, v_{2}$ and $v_{3}$ be any vertices in $G$ such that $v_{1}$ adjacent to $v_{2}$ and $v_{3}$. Let $\operatorname{deg}_{\text {in }}\left(v_{1}\right)=i$ in $H$. Therefore, $\operatorname{deg}_{\text {in }}\left(v_{2}\right)=i$ or $i-1$ or $i+1$ and $\operatorname{deg}_{\text {in }}\left(v_{3}\right)=i-1$ or $i$ or $i+1$.

Case 1. $\operatorname{deg}_{\text {in }}\left(v_{2}\right)=i$. If $\operatorname{deg}_{\text {in }}\left(v_{3}\right)=i-1$ or $i$ or $i+1$ then $v_{2}$ and $v_{3}$ are adjacent which contradicts that $G$ is a cycle.

Case 2. $\operatorname{deg}_{\text {in }}\left(v_{2}\right)=i-1$. If $\operatorname{deg}_{\text {in }}\left(v_{3}\right)=i$ or $i-1$, then $v_{2}$ and $v_{3}$ are adjacent which contradicts that $G$ is a cycle. Therefore, $\operatorname{deg}_{i n}\left(v_{3}\right)=i+1$.

Case 3. $\operatorname{deg}_{\text {in }}\left(v_{2}\right)=i+1$. If $\operatorname{deg}_{\text {in }}\left(v_{3}\right)=i$ or $i+1$, then $v_{2}$ and $v_{3}$ are adjacent which contradicts that $G$ is a cycle. Therefore, $\operatorname{deg}_{\text {in }}\left(v_{3}\right)=i-1$.

Hence the graph $H$ has different injective degrees which contradicts that any graph with $n$ vertices has at least two vertices of the same Inj-degree. Therefore, any cycle $C_{n}$ is not $I E$-graph.

Lemma 34 Any bipartite graph is not IE-graph.

Proof. Suppose, to the contrary, that $G$ is a bipartite graph such that $G$ is $I E$-graph. Therefore, there exists a graph $H$ such that $I E(H) \cong G$. Let $v_{1}, v_{2}$ and $v_{3}$ be any vertices in $G$ such that $v_{1}$ adjacent to $v_{2}$ and $v_{2}$ adjacent to $v_{3}$. Let $\operatorname{deg}_{\text {in }}\left(v_{1}\right)=i$ in $H$. Therefore $\operatorname{deg}_{\text {in }}\left(v_{2}\right)=i-1$ or $i$ or $i+1$.

Case 1. $\operatorname{deg}_{\text {in }}\left(v_{2}\right)=i$. If $\operatorname{deg}_{\text {in }}\left(v_{3}\right)=i-1$ or $i$ or $i+1$ then $v_{2}$ and $v_{3}$ are adjacent in $G$. So, $G$ contains an odd cycle which contradicts that $G$ is a bipartite graph.

Case 2. $\operatorname{deg}_{\text {in }}\left(v_{2}\right)=i-1$. If $\operatorname{deg}_{\text {in }}\left(v_{3}\right)=i$ or $i-1$, then $v_{2}$ and $v_{3}$ are adjacent in $G$. So, $G$ contains an odd cycle which contradicts that $G$ is a bipartite graph. Therefore, $\operatorname{deg}_{i n}\left(v_{3}\right)=i+1$. 
Case 3. $\operatorname{deg}_{\text {in }}\left(v_{2}\right)=i+1$. If $\operatorname{deg}_{\text {in }}\left(v_{3}\right)=i$ or $i+1$, then $v_{2}$ and $v_{3}$ are adjacent in $G$. So, $G$ contains an odd cycle which contradicts that $G$ is a bipartite graph. Therefore, $\operatorname{deg}_{\text {in }}\left(v_{3}\right)=i-1$.

Hence the graph $H$ has different injective degrees which contradicts that any graph with $n$ vertices has at least two vertices of the same Inj-degree. Therefore, any bipartite graph is not $I E$-graph.

Theorem $35 A$ connected graph $G$ is IE-graph if $G$ is a chain of complete graphs.

Proof. Suppose that $G$ is a connected $I E$-graph. Then there exists a graph $H$ such that $I E(H) \cong G$. Each $\left\langle S_{i}\right\rangle$ is complete in $I E(H)$ and there are $S_{i} \cap S_{i+1}$ common vertices between $\left\langle S_{i}\right\rangle$ and $\left\langle S_{i+1}\right\rangle$. Therefore, $G$ is a chain of complete graphs.

\section{Conclusion}

In this paper, we introduced the injective equitable graph of a graph $\operatorname{IE}(G)$ and the injective equitable graph $I E$-graph. We defined these graphs and presented some of their properties. Also, we found the Inj-equitable graph of some graph's families. The degree injective equitable set, the degree injective equitable number of a graph and the lower degree injective equitable number are defined. Relations on those parameters in terms of maximum vertex degree, minimum vertex degree, diameter and clique number of the injective equitable graph are established. The connectedness of $I E(G)$ and relations between the $\operatorname{IE}(G), \operatorname{Con}(G)$ and $G^{e}$ are studied. In the last of this paper, the sufficient condition for a connected graph $G$ to be $I E$-graph is presented.

\section{References}

[1] Alwardi, A., Alqesmah, A. and Rangarajan, R. (2016) Independent Injective Domination of Graphs. International Journal of Applied Mathematics and Mechanics, 3, 142-151.

[2] Balakrishnan, R. and Ranganathan, K. (2000) A Textbook of Graph Theory. Springer-Velag, New York. https://doi.org/10.1007/978-1-4419-8505-7

[3] Chartrand, G. and Lesniak, L. (2005) Graphs and Diagraphs. 4th Edition, CRC press, Boca Raton.

[4] Harary, F. (1969) Graphs Theory. Addison-Wesley, Reading Mass. https://doi.org/10.21236/AD0705364

[5] Alwardi, A., Arsic, B., Gutman, I. and Soner, N.D. (2012) The Common Neighborhood Graph and Its Energy. Iranian Journal of Mathematical Sciences and Informatics, 7, 1-8.

[6] Dharmalingam, K.M. (2012) Equitable Graph of a Graph. Proyecciones Journal of Mathematics, 31, 363-372. https://doi.org/10.4067/S0716-09172012000400005

[7] Alkenani, A., Alashwali, H. and Muthana, N. (2016) On the Injective Equitable Domination of Graphs. Applied Mathematics, 7, 2132-2139.

https://doi.org/10.4236/am.2016.717169 